\title{
Homer1 promotes dendritic spine growth through ankyrin-G and its loss reshapes the synaptic proteome
}

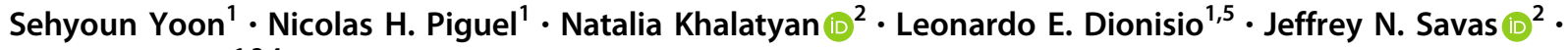 \\ Peter Penzes $\mathbb{D}^{1,3,4}$
}

Received: 22 February 2020 / Revised: 24 November 2020 / Accepted: 7 December 2020 / Published online: 4 January 2021

(c) The Author(s), under exclusive licence to Springer Nature Limited part of Springer Nature 2021. This article is published with open access

\begin{abstract}
Homer1 is a synaptic scaffold protein that regulates glutamatergic synapses and spine morphogenesis. HOMERl knockout (KO) mice show behavioral abnormalities related to psychiatric disorders, and HOMERl has been associated with psychiatric disorders such as addiction, autism disorder (ASD), schizophrenia (SZ), and depression. However, the mechanisms by which it promotes spine stability and its global function in maintaining the synaptic proteome has not yet been fully investigated. Here, we used computational approaches to identify global functions for proteins containing the Homer1-interacting PPXXF motif within the postsynaptic compartment. Ankyrin-G was one of the most topologically important nodes in the postsynaptic peripheral membrane subnetwork, and we show that one of the PPXXF motifs, present in the postsynaptically-enriched $190 \mathrm{kDa}$ isoform of ankyrin-G (ankyrin-G 190), is recognized by the EVH1 domain of Homer1. We use proximity ligation combined with superresolution microscopy to map the interaction of ankyrin-G and Homer1 to distinct nanodomains within the spine head and correlate them with spine head size. This interaction motif is critical for ankyrin-G 190's ability to increase spine head size, and for the maintenance of a stable ankyrin-G pool in spines. Intriguingly, lack of Homer1 significantly upregulated the abundance of ankyrin-G, but downregulated Shank3 in cortical crude plasma membrane fractions. In addition, proteomic analysis of the cortex in HOMERI KO and wild-type (WT) mice revealed a global reshaping of the postsynaptic proteome, surprisingly characterized by extensive upregulation of synaptic proteins. Taken together, we show that Homer1 and its protein interaction motif have broad global functions within synaptic protein-protein interaction networks. Enrichment of disease risk factors within these networks has important implications for neurodevelopmental disorders including bipolar disorder, ASD, and SZ.
\end{abstract}

Supplementary information The online version of this article (https:// doi.org/10.1038/s41380-020-00991-1) contains supplementary material, which is available to authorized users.

$\triangle$ Peter Penzes

p-penzes@northwestern.edu

1 Department of Physiology, Northwestern University Feinberg School of Medicine, Chicago, IL 60611, USA

2 Department of Neurology, Northwestern University Feinberg School of Medicine, Chicago, IL 60611, USA

3 Department of Psychiatry and Behavioral Sciences, Northwestern University Feinberg School of Medicine, Chicago, IL 60611, USA

4 Northwestern University, Center for Autism and Neurodevelopment, Chicago, IL 60611, USA

5 Present address: Neuroscience Interdepartmental Program, David Geffen School of Medicine, University of California, Los Angeles, CA 90095, USA

\section{Introduction}

The structural plasticity of dendritic spines is governed by several processes including spine formation, maturation, stabilization, remodeling, and elimination. Changes in spine number and morphology, along with the glutamate receptor content of synapses, contribute to functional connectivity in synaptic circuits [1]. Altered spine density in cortical pyramidal neurons has been observed in postmortem studies of patients with schizophrenia (SZ), bipolar disorder (BD), autism spectrum disorder (ASD), and intellectual disability (ID) [2-5]. Thus, dendritic spine abnormalities on cortical pyramidal neurons have emerged as key cellular substrates in the pathogenesis of several psychiatric disorders $[6,7]$.

The Homer family consists of three members in mammals, Homer1, Homer2, and Homer3, all of which are postsynaptic density scaffolding proteins highly expressed in the central nervous system. Homer1 protein regulates glutamatergic synapses and spine morphogenesis [8-10]. 
HOMERI is associated with ASD [11], SZ [12], and depression [13] in human cohorts and, HOMERI KO mice show relevant behavioral abnormalities related to the above psychiatric disorders $[14,15]$. The Homer1 EVH1 domain interacts with the PPXXF motif [16] and the longer isoforms of Homer1b/c include the coiled-coiled C-terminus which mediates its multimerization [17]. Multimerized Homerlb/c forms a mesh-like matrix structure in dendritic spines and serves as an assembly platform for Shank proteins to positively regulate spine morphology [18]. However, the complete list of Homer1-interacting proteins has not yet been identified and the mechanisms by which Homer1 regulates spine structure are not fully understood. To identify novel Homerl interaction partners we performed an in silico screen for proteins encoded by neuropsychiatric risk genes with a PPXXF motif, and identified the synaptic scaffold protein ankyrin-G.

Genetic studies have shown that synaptic genes are key factors in the pathogenesis of neuropsychiatric disorders $[19,20]$. Among these, common variants at the ANK3 gene locus are one of the most strongly associated risk factors for BD in genome-wide association studies (GWAS) [21, 22]. The isoforms of $190 / 270 / 480 \mathrm{kDa}$ of ankyrin-G express in the brain [23], and the largest $270 / 480 \mathrm{kDa}$ isoforms have well-characterized roles at the axon initial segment (AIS) and nodes of Ranvier [24]; however, the role of the $190 \mathrm{kDa}$ isoform has been mostly studied in the dendritic spines [2528]. Even though the major isoforms of ankyrin-G share four conserved domains [29], brain-specific expressing exons generate unique properties of ankyrin- $G$ isoforms [30]. Intriguingly, allelic association tests supported that single nucleotide polymorphisms (SNPs) into ankyrin-G 190 specific exons were associated with SZ and BD [3133]. However, the mechanism through which ankyrin-G 190 regulates these processes is elusive, largely due to the lack of information on its protein interactome and the signaling networks regulating ankyrin-G in spines.

Here, we identified a PPXXF domain present in ankyrin-G 190, which is recognized by Homer1, and confirmed the interaction between ankyrin-G and Homer1 by immunoprecipitation and Proximity Ligation Assay (PLA). Using superresolution microscopy, we find that the interaction of ankyrin$\mathrm{G}$ and Homer1 occurred in distinct nanodomain structures within the spine head and that the number of both ankyrin-G and Homerlb/c puncta correlates with the head size of mushroom spines. This interaction was reduced by disrupting the PPXXF motif through single amino acid modifications. We have shown that ankyrin-G and Homer1, both neuropsychiatric disorder factors, interact in a complex to regulate spine morphology and to modulate the steady-state levels of ankyrin-G in the spine head. In addition, a comparison of protein abundances in the crude plasma membrane (P2) fraction from the cortex of HOMERI KO and wild-type
(WT) mice demonstrated global proteomic alterations, characterized by extensive upregulation of synaptic proteins. Taken together, these findings demonstrate that the ankyrin-G 190 and Homer1 interaction regulates spine stability, revealing novel mechanisms underlying spine structure that may be relevant to neuropsychiatric disease.

\section{Materials and methods}

\section{Plasmids and antibodies}

3XHA-ankyrin-G (EX-Mm25668-M06) was purchased from GeneCopoeia. Three domain fragments of ankyrin-G (amino acids 1-807, 808-1475, 1476-1961) were amplified from 3XHA-ankyrin-G. GFP-tagged ankyrin-G was amplified from 3XHA-ankyrin-G, and subcloned into pEGFP-N2 (\#6801-1; Clontech) using the Infusion ligation independent cloning kit (Clontech). The detailed information was described previously [26, 27]. 3XHA-ankyrin-G P1606L, F1609R and GFP-ankyrin-G F1609R were generated using QuickChange Site-Directed Mutagenesis Kit (Agilent) as per the manufacturer's instructions. Homer1c was amplified from pmEmerald-Homer1c (Addgene; \#54120) and subcloned into pEZ-M12 to generate 3XFlagHomer1c using the Infusion ligation independent cloning kit (Clontech). The following primary antibodies were used: mouse ankyrin-G (Neuromab; N106/36), rabbit ankyrin-G (SantaCruz; sc-28561), rabbit Homer1b/c (Antibodiesonline; ABIN-1742339), mouse Homer1b/c (SantaCruz; sc25271), mouse Flag (Sigma; F1804), mouse HA (Abcam; ab130275), rabbit Flag (Sigma; F7425), rabbit HA (Enzo; ADI-MSA-106), mouse PSD-95 (NeuroMab; K28/43), mouse Na K ATPase $\alpha 1$ (Novus; NB-300-146), mouse $\beta$-actin (Sigma; A2228), mouse Flag-488 (Abcam; ab117505), rabbit HA-488 (Abcam; ab117505), rabbit HA568 (Synaptic System, 245 003C3), goat mCherry (SICGEN; AB0040), and rabbit GAPDH (Cell signaling; 5174).

\section{Co-immunoprecipitation assays}

Six cortical hemispheres were dissected from 16-week-old mice and were homogenized in cold sucrose buffer $(20 \mathrm{mM}$ HEPES pH 7.4, $320 \mathrm{mM}$ sucrose, $5 \mathrm{mM}$ EDTA) with protease inhibitor cocktail (Roche). Homogenates were centrifuged at $3000 \times g$ for $20 \mathrm{~min}$ at $4{ }^{\circ} \mathrm{C}$ to pellet nuclei. The supernatant (S1; cytosol/membranes) was then centrifuged at $38,000 \times \mathrm{g}$ for $30 \mathrm{~min}$ at $4{ }^{\circ} \mathrm{C}$ to obtain crude synaptosomes (P2) from pellets and cytosol (S2) from the supernatant. $\mathrm{P} 2$ was resuspended in $0.5 \mathrm{ml}$ of tris buffer (per cortex) (20 mM Tris pH 7.4, $150 \mathrm{mM} \mathrm{NaCl}, 1 \mathrm{mM}$ EGTA with protease inhibitors). We then added $0.5 \mathrm{ml}$ of $2 \mathrm{x}$ Triton buffer (per cortex) (20 mM Tris pH 7.4, $150 \mathrm{mM}$ 
$\mathrm{NaCl}, 1 \mathrm{mM}$ EGTA, 2\% Triton $\mathrm{X}-100$ with protease inhibitors) to $\mathrm{P} 2$ and rotated it for $1 \mathrm{~h}$ at $4{ }^{\circ} \mathrm{C}$ to solubilize. The samples were centrifuged at $16,000 \times g$ for $10 \mathrm{~min}$ at $4{ }^{\circ} \mathrm{C}$. Triton-insoluble pellets were solubilized by adding $100 \mu \mathrm{l}$ of SDS buffer (20 mM Tris $\mathrm{pH} 7.4,150 \mathrm{mM} \mathrm{NaCl}, 1 \mathrm{mM}$ EGTA, $1 \%$ SDS with protease inhibitors) and incubating at $37^{\circ} \mathrm{C}$ for $20 \mathrm{~min}$. The samples were centrifuged at $16,000 \times$ $g$ for $10 \mathrm{~min}$ at $4{ }^{\circ} \mathrm{C}$ again. The final supernatant contains PSD-enriched synaptosomal fraction. For the immunoprecipitation, PSD-enriched samples were diluted in tris buffer (the final concentration per each antibody: $150 \mu \mathrm{g} / 1.0 \mathrm{ml}$ ).

For assays of overexpressed ankyrin-G and Homer1c constructs, HEK293T cells (ATCC; CRL-11268) were transfected with 3XHA-ankyrin-G and 3XFlag-Homer1c using PEI transfection reagent (Sigma) in two 100-mm dishes per each construct. The detailed information for immunoprecipitation was described previously [26, 27]. For the western blotting, VeriBlot for IP Detection Reagent (Abcam; ab131366) was used to avoid overlapping the heavy chain bands with 3 XFlag-Homer1c. The membrane was blocked with Protein-Free T20 (TBS) Blocking buffer (Pierce; \#37571), and primary and secondary antibodies were diluted in the same buffer.

\section{Neuronal cell culture and transfection}

Dissociated primary cortical cultured neurons were prepared from P0 C57BL/6J (The Jackson Laboratory). Brains were dissected in ice-cold Leibowitz's L-15 media with penicillin/streptomycin, and cortical tissue isolated, digested with $0.25 \%$ trypsin-EDTA solution at $37^{\circ} \mathrm{C}$ for $10 \mathrm{~min}$, and mechanically dissociated in high-glucose Dulbecco's Modified Eagle Medium (DMEM) supplemented with 10\% FBS, $1.4 \mathrm{mM} \mathrm{L-glutamine,} \mathrm{and} 6.0 \mathrm{~g} / \mathrm{L}$ glucose. The detailed information was described previously [26, 27]. All procedures were in compliance with the National Institutes of Health standards and were approved by Northwestern University's Animal Care and Use Committee.

\section{Confocal microscopy}

Immunostained neuronal images were obtained with a Nikon $\mathrm{C} 2+$ confocal microscope. Confocal images were taken using the $63 \mathrm{x}$ oil-immersion objective $(\mathrm{NA}=1.4)$ as z-series of $8-10$ images, taken at $0.4 \mu \mathrm{m}$ intervals, with $1024 \times 1024$ pixel resolution. Detector gain and offset were adjusted in the channel of cell fill (mCherry) to include all spines and enhance edge detection.

\section{Immunocytochemistry}

By using 4\% paraformaldehyde in PBS, cells were fixed for $10 \mathrm{~min}$ at $4{ }^{\circ} \mathrm{C}$. PBS washing for $5 \mathrm{~min}$ (three times), fixed neurons were permeabilized and blocked in PBS containing $0.3 \%$ Triton-X-100 and $1 \%$ bovine serum albumin (BSA) for $1 \mathrm{~h}$ at room temperature. Primary antibodies were added in PBS containing $0.3 \%$ Triton-X-100 and $1 \%$ BSA overnight at $4^{\circ} \mathrm{C}$, followed by $10 \mathrm{~min}$ washes in PBS (three times). Secondary antibodies were incubated for $1 \mathrm{~h}$ at room temp in $0.3 \%$ Triton-X-100 and $1 \%$ BSA in PBS. PBS washing for 5 min (three times) was performed before coverslips were mounted using Fluorescent Mounting Medium (Invitrogen).

\section{SIM imaging and analysis}

With EM gain and no binning, the acquisition was set to $10 \mathrm{MHz}, 14$ bits. The EM gain multiplier restrained below 300 , and auto exposure was set between 100-300 ms and. Within the first quarter of the scale $(<4000)$, the laser power was adjusted to keep LUTs. Imaging and reconstruction parameters were determined with the assistance of the expertise in the Center for Advanced Microscopy at Northwestern University. The single-plane where the spine head was in focus, based on the cell fill, was chosen for analysis. Each spine head was outlined using the Image $\mathbf{J}$ software manually in the channel of the cell fill to detect the area. Within the spine head, ankyrin-G and Homer1b/c puncta were outlined manually, and the size was recorded. A $100 \mu \mathrm{m}$ dendritic region was selected, and puncta counts were made; puncta smaller than $0.006 \mu \mathrm{m}^{2}$ were excluded from the analysis. To present more informative images, z-series images were reconstituted by Imaris v9.1.2 (OXFORD instruments).

\section{Proximity ligation assay (PLA)}

Cortical neurons were transfected with pmCherry-C1 (Clontech, \#632524) and 3XHA-ankyrin-G at DIV21. The transfected cells for three days were fixed with $4 \%$ paraformaldehyde in PBS for $10 \mathrm{~min}$ at $4{ }^{\circ} \mathrm{C}$. For the negative control, PLA experimented on only mCherry transfected cultured neurons. With 3XFlag-Homer1c and 3XHAankyrin-G, COS7 cells were transfected using lipofectamine 2000 reagent. The transfected cells were fixed at $24 \mathrm{~h}$ posttransfection. Rabbit 568 conjugated-HA and mouse 488 conjugated-Flag antibodies were treated to confirm the transfection of constructs into the cells. Duolink ${ }^{\mathrm{TM}}$ In Situ Detection Reagents FarRed (Sigma, DUO92013) was used to detect the PLA signal.

\section{Fluorescent recovery after photobleaching (FRAP)}

Cortical neurons for FRAP experiments were grown on $18 \times$ $18 \mathrm{~mm}$ coverslip and transfected with GFP alone, GFPankyrin-G WT, or GFP-ankyrin-G F1609R. Coverslip was placed in a round bath chamber (Warner Instruments; RC41LP) and neurons were imaged with a C2 confocal 
microscope (Nikon) in a $\mathrm{CO} 2$ incubator (TOKAI HIT; UNIVD35). The size of spine heads in GFP-ankyrin-GF1609R transfected neurons generally was downsized, and we decided to match the spine head size from each group. Images were captured with an EMCCD camera of $512 \times 512$ pixel resolution every $10 \mathrm{~s}$ for $200 \mathrm{~s} .100 \%$ laser power pulses of $1 \mathrm{~ms}$ (2 iterations) were used to bleach GFP clusters. Fluorescence intensity of bleached GFP clusters was measured and normalized to the fluorescence of an unbleached region to correct for photobleaching. These values were normalized to the mean of the 5 frames prior to bleaching, and the lowest value in the dataset was subtracted from all values. Recovery data points were then fitted to a one-phase association exponential in GraphPad Prism8. The mobile fraction was calculated as an average of the plateaued fluorescence level and expressed as a percentage of the pre-bleached level. We determined the mobile fraction by calculating the ratio of fluorescence intensity between the end of the time-lapse recordings $\left(\mathrm{F}_{\text {end }}\right)$ and the initial intensity before the bleaching event $\left(\mathrm{F}_{\text {initial }}\right)$, corrected by the experimental bleach value $\left(\mathrm{F}_{0}\right)$ and expressed as a percentage.

\section{Quantitative proteomics and analysis}

Homer1 heterozygous mice (The Jackson Laboratory; \#023312) were mated with the same heterozygous mice to maintain the colonies. Littermates that five HOMERl KO mice and five WT mice (all females) from 4 cohorts were used for the proteomic analysis. The mice were housed with mixed-genotype home cages together until testing. Within an SPF barrier area mice were maintained on a 12:12 h light/dark schedule in an air-conditioned room, under constant conditions of temperature and humidity. Food and tap water (membrane filter purified and autoclaved water) were provided ad libitum. All experiments were performed in accordance with the Institutional Animal Care and Use Committee at Northwestern University.

3-week-old mouse cortex was dissected and homogenized in sucrose buffer (20 mM HEPES pH 7.4, $320 \mathrm{mM}$ sucrose, $5 \mathrm{mM}$ EDTA with Roche protease inhibitors) and centrifuged at $3000 \times g$ for $20 \mathrm{~min}, 4^{\circ} \mathrm{C}$. The collected supernatant was centrifuged further at $16,000 \times g$ for $30 \mathrm{~min}$ at $4{ }^{\circ} \mathrm{C}$ to obtain crude synaptosomes (P2) and collect supernatant for cytosolic proteins (S2). Pellets were resuspended with binding buffer $(50 \mathrm{mM}$ Tris- $\mathrm{HCl} \mathrm{pH} 7.5,1 \%$ triton-X-100, $150 \mathrm{mM} \mathrm{NaCl}, 1 \mathrm{mM}$ EDTA, $1 \mathrm{mM}$ AEBSF with protease inhibitor cocktail and phosphatase inhibitor) and solubilized for $1 \mathrm{~h}$ at $4{ }^{\circ} \mathrm{C}$. The detailed information for Tandem Mass Tag-liquid chromatography/mass spectrometry (TMT-LC/MS) was described previously [34].

The normalized average intensity values for proteins from Census were used to calculate the fold change WT versus KO. The values were standardized to the mean of the five WT samples, and the fold change could then be calculated as the mean of the KO standardized values. $P$ values were calculated by one-tailed Student's $t$ test. For GO analysis, the list of significantly upregulated or downregulated proteins was queried against all proteins quantified in the dataset using a statistical overrepresentation test of the DAVID Bioinformatics Resources (v6.8). Biological processes which were a subset of a larger group that was also statistically significant defaulted to the more encompassing annotation and its associated $P$-value. For STRING analysis, 82 regulated proteins in postsynaptic density (PSD) from the cortex of HOMERI KO mice were loaded into multiple proteins option of STRING (v11.0) and analyzed setting with all interaction sources and medium confidence $(0.400)$ score. The data were exported and the list of interactors was sorted PSD proteins and ASD risk factors out again. To generate a representative interacting network in PSD, 63 of sorting proteins were analyzed by Cytoscape (v3.7.1).

\section{Blinding and statistical analysis}

Data from spine morphology and dynamics, protein localization, and PLA analysis in pyramidal neurons (in vitro) were analyzed under blinded conditions (coverslip identity hidden and raw data pooled during quantification). Fractionation, immunoprecipitation, western blotting, and MS experiments were not blinded. Cells of visibly poor health (i.e., blebs, broken dendrites, poor expression, etc.) were excluded from quantification. No animals were excluded from the analysis, and no method of animal randomization was employed. Sample sizes were on average between 9 and 13 cells for endogenous analysis and between 16 and 18 cells for overexpression analysis to account for cellular diversity. Five animals per genotype were used for western blotting and MS experiments, which was sufficient for statistical significance.

GraphPad Prism 8 was used for all statistical tests. Twosample comparisons were performed using two-tailed unpaired Student's $t$ test or two-tailed non-parametric Spearman correlation, and multiple comparisons were made using one-way ANOVA followed by non-parametric statistical analysis or two-way ANOVA followed by a Bonferroni test. Statistical details are given in each figure legend. Bar graphs are displayed as mean \pm SEM. $P$ values $<0.05$ were considered statistically significant.

\section{Results}

\section{Proteins containing the Homer1-binding PPXXF motif are abundant within postsynaptic and disease networks}

The PPXXF motif present in some proteins known to interact with Homer's EVH1 ligand motif is important for protein- 
protein interactions [16]. To determine whether Homer1binding proteins are enriched in the PSD, we retrieved all PPXXF-containing proteins identified in UniProt $(n=1995)$ with the aid of the MOTIF search and performed a gene set analysis with genes encoded by PSD proteins $[35,36]$. We found a subset of genes encoding PPXXF-containing proteins that have been reported to be localized to PSDs $(n=227$, $11.4 \%$, Fig. 1a). To evaluate the conservation of the PPXXF motif across species within this PSD subset, we examined the sequence of the motif in human, bovine, mouse, and rat (Supplementary Table 1), and 150 proteins were confirmed to have a conserved PPXXF motif. Gene ontology (GO) analysis revealed this PPXXF-PSD gene set was highly enriched for specific biological processes including proteins regulating glutamatergic synapses, retrograde endocannabinoid signaling and long-term potentiation (Fig. 1b). To determine whether PPXXF motif-containing proteins in the PSD a role in neuropsychiatric disease, we again performed gene set analysis with bipolar disorder, autism and schizophrenia-associated gene sets. We found a significant enrichment of PPXXF motifcontaining proteins in genome-wide significant loci from $\mathrm{BD}$ GWAS (7/120) and de novo variants associated with ASD and SZ (30/1167). The enrichment of PSD-PPXXF motif-containing proteins within neuropsychiatric disease risk factors suggests proteins containing this motif may play a role in pathophysiology (Fig. 1c, d). To determine the functional relationship between these risk genes we seeded a proteinprotein interaction (PPI) network with the 33 proteins associated with neuropsychiatric disease. We found that the majority of PPXXF motif-containing psychiatric risk factors in PSDs are physically connected (20 nodes, 43 edges) (Fig. 1e; Supplementary Fig. 1). By performing network analysis, we found that Cacnalc and Shank3 were the central hubs and ankyrin-G is one of the most topologically important nodes in the peripheral membrane as an unknown interactor of Homer.

We found two PPXXF motifs in ankyrin-G (Fig. 1f, g). One was within the "regulatory domain", present in the $190 \mathrm{kDa}$ isoform, but not 270 and $480 \mathrm{kDa}$ isoforms (ankyrin-G 190: 1605-1609). A second motif was within a region present in the giant ankyrin-G forms, including 270 and $480 \mathrm{kDa}$ forms, but not the $190 \mathrm{kDa}$ isoform (ankyrin-G 270 and 480: 2187-2191).

\section{Ankyrin-G 190 and Homer1b/c interact in nanodomains within dendrites and spines}

Because here we are investigating postsynaptic interactions within spines, and both Homerl and ankyrin-G 190 are enriched in spines, we decided to focus on the interaction of ankyrin-G 190 with Homer1, in postsynaptic regions. In order to confirm the spatial co-distribution of ankyrin-G and Homerlb/c and investigate the interaction of these proteins in vivo, we performed subcellular fractionation of mouse cortex by ultracentrifugation and detergent solubilization. Both ankyrin-G 190 and Homer1b/c were highly expressed in a PSD-enriched fraction (Fig. 2a). We then used this PSDenriched fraction for coimmunoprecipitation experiments and showed that ankyrin-G 190 , but not 270 , robustly coimmunoprecipitated with Homer1b/c from this fraction (Fig. 2b). To determine the interaction sites between ankyrin-G and Homer1c, we conducted immunoprecipitation experiments with three HA-tagged truncated ankyrin-Gs (amino acids 1-807, 808-1475, 1476-1961) and Flag-tagged Homer1c. As predicted, we found that Homerlc robustly coimmunoprecipitated with the regulatory domain (1476-1961) of ankyrin-G 190 (Fig. 2c). However, we detected a weaker interaction with the ankyrin-repeat domain of ankyrin-G as well (1-807) (Supplementary Fig. 2). One previous study showed that a point mutation (proline to leucine or phenylalanine to arginine) of the Homer-binding motif impaired interaction with diacylglycerol lipase type- $\alpha$ [37]. To determine the role of the ankyrin-G PPXXF motif in the Homerlc interaction, PPXXF mutants (Pro1606Leu and Phe1609Arg) of ankyrin-G were produced and coimmunoprecipitated after overexpression in HEK293T cells. Point mutations in the PPXXF Homer1-binding motif of ankyrin-G resulted in the attenuation of Homer1c interaction (Fig. 2d). To test the interaction of ankyrin-G and Homerlb/c, in situ PLA was employed. This technique labels interacting proteins that fall within a defined, 16-nanometer distance (as calculated from the number of nucleotides in the attached DNA arms [38]), a strong indicator of direct protein-protein interaction or complex formation (Fig. 2e). Despite robust interaction with the native ankyrin-G, the PLA signal was significantly decreased in cells expressing Homer1 and PPXXF mutated ankyrin-G (Fig. 2f). Colocalization and the subcellular compartments of Homerlb/c and ankyrin-G complexes were assessed using PLA in primary cortical neurons, confirming an interaction within spine heads (Fig. 2g, h). Taken together, these data indicate that ankyrin-G 190's binding to Homer1 through its PPXXF motif strongly modulates their interaction, while an additional binding site in the ankyrin-repeat region, present in all ankyrin-G isoforms, may also affect their interaction.

Because both ankyrin-G and Homerlb/c have been detected in the PSD by proteomics, we hypothesized that their interaction might have a role in regulating spine morphology [36]. To understand how the localization of ankyrin-G and Homer1b/c nanodomains correlates with spine morphology, we imaged mouse primary cortical neurons stained for endogenous ankyrin-G and Homer1b/c by SIM microscopy and analyzed their subcellular distribution (Fig. 2i, j). Manders' colocalization coefficients showed that ankyrin-G colocalizes with Homer1b/c in the dendritic shaft and spine heads. Interestingly, ankyrin-G/ Homer1b/c overlapping nanodomains were largely 
a

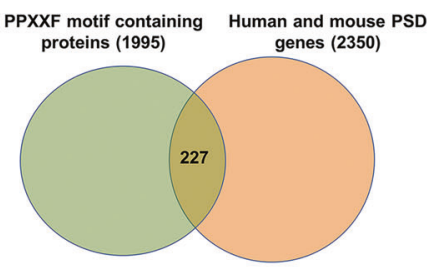

b

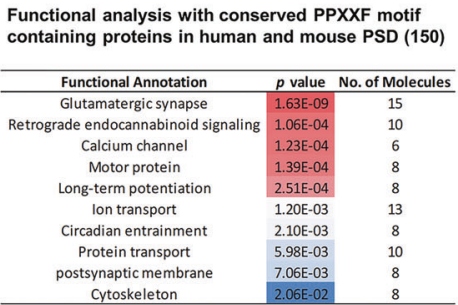

c

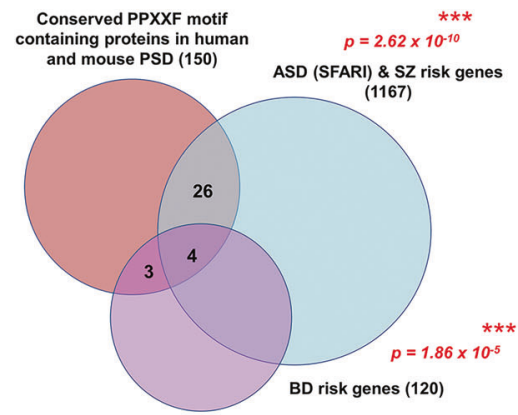

d

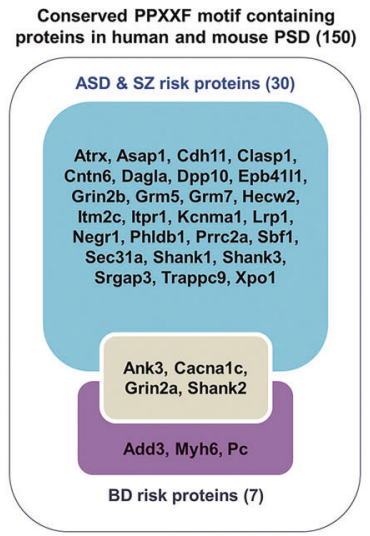

e

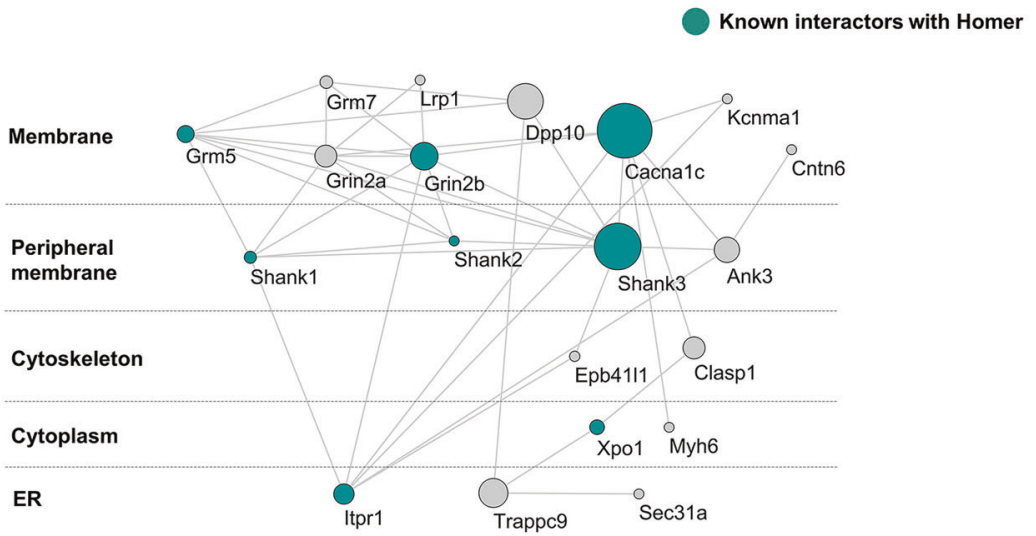

f

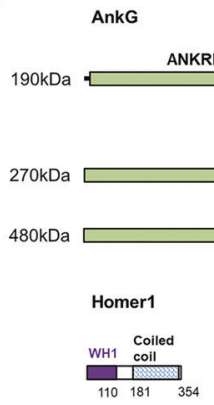

Fig. 1 Multiple postsynaptic psychiatric risk factors include a Homer1-binding motif. a Diagram of PPXXF motif-containing proteins in PSD. b Functional analysis of conserved (in human, bovine, mouse, and rat) PPXXF motif-containing proteins in PSD. c Enrichment of BD, ASD, and SZ risk factors (identified through GWAS, SFARI gene archive and de novo studies, respectively) among PPXXF motif-containing proteins in the combined human and mouse PSD. $* * * p<0.001$; hypergeometry test. d Diagram of PPXXF motifcontaining proteins in the PSD is encoded by psychiatric risk genes. $\mathbf{e}$ Protein interaction network generated from the list of PPXXF motifcontaining PSD proteins is encoded by psychiatric risk genes in $\mathbf{d}$. The

localized in the spine head (Fig. 2k). Ankyrin-G or Homer1b/c puncta were observed in 58.7 and $81.5 \%$ of spines, respectively. Both ankyrin-G and Homerlb/c were observed in $51.2 \%$ of spines (Fig. 21). Spine heads expressing ankyrin- $G$ are significantly larger than spine heads without ankyrin-G (Fig. 2m, n), as previously reported [28]. The presence of Homer1b/c in spine heads representative majority of a subnetwork is visualized and annotated by Cytoscape. The size of node indicates betweenness centrality. Known interactors with Homer1/2/3 from BioGrid and text mining were colored emerald. Edges indicate known and predicted protein-protein interactions, including experimental data from the STRING database. f Schematic representation of the Homerl recognition motifs in ankyrin$\mathrm{G}(190,270$, and $480 \mathrm{kDa})$ isoforms. g Amino acid sequence alignment of human, bovine, mouse, and rat ankyrin-G regions containing the Homer-binding motif PPXXF. Sequence alignments were performed by Clustal Omega. SFARI: Simons Foundation Autism Research Initiative.

was also significantly correlated to larger spine head size (Fig. 2o). Homer1b/c clusters had a mean area of $0.056 \pm$ $0.003 \mu \mathrm{m}^{2}$, and this area correlated to the size of ankyrin-G nanodomains and spine head area (Fig. 2p). Taken together, the size of Homer1b/c clusters in spine head is positively correlated to the number and size of ankyrin-G nanodomains, and spine head size. 
a

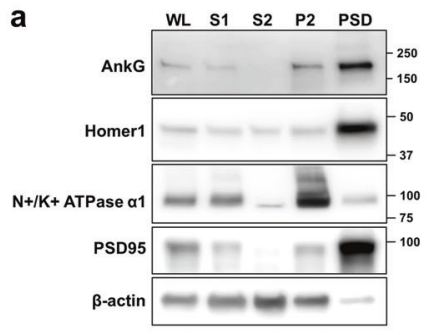

b

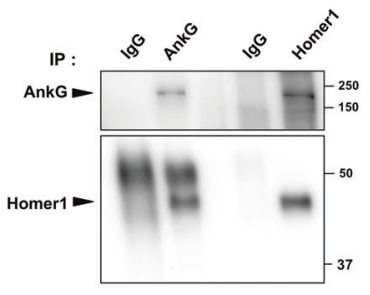

e

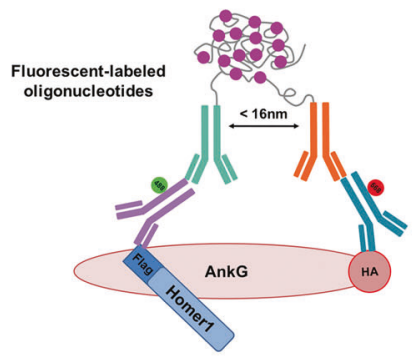

C

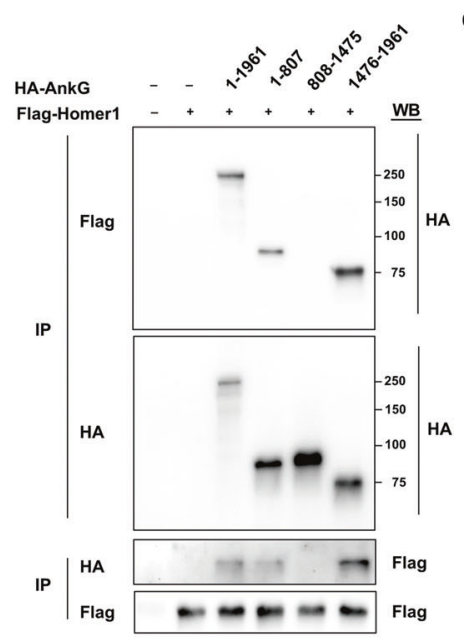

d
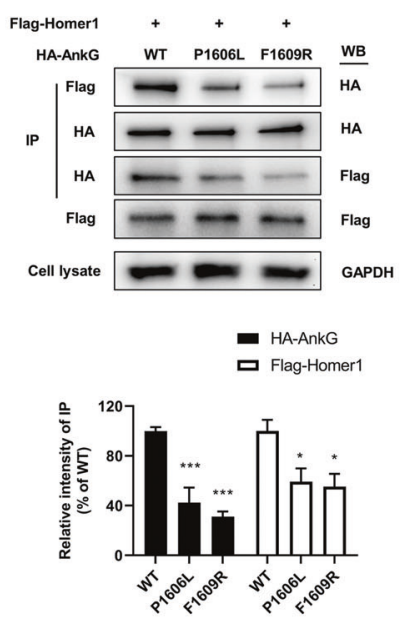

f

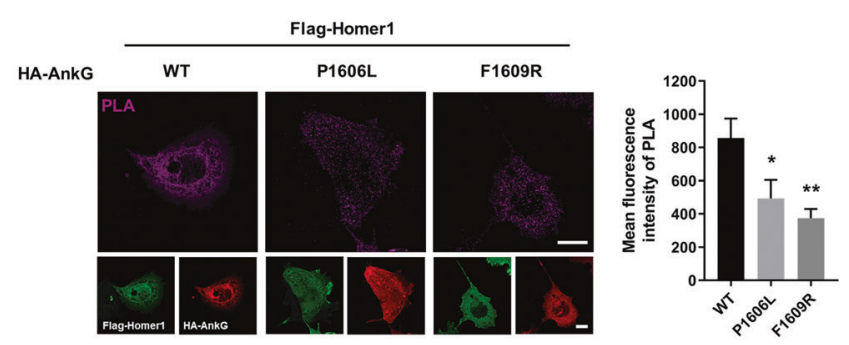

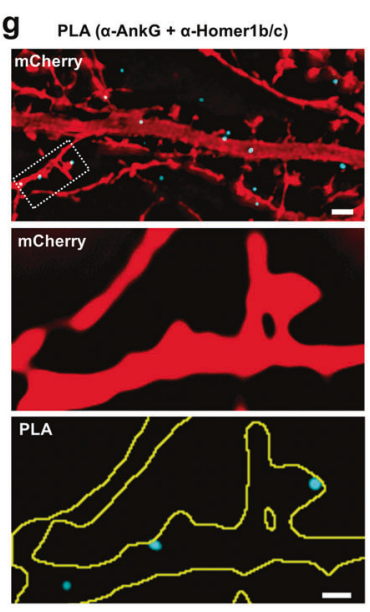

h

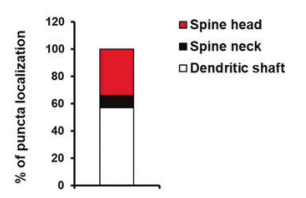

i AnkG/Homer1b/c Highlighting Colocalization

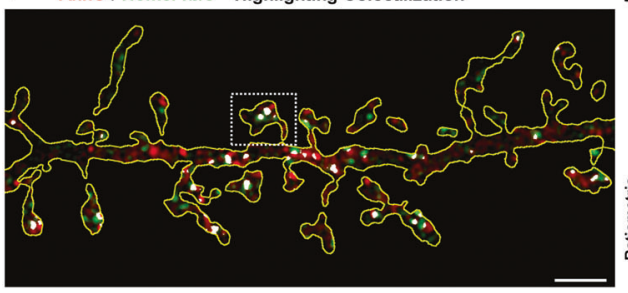

$$
\text { k }
$$

Number of colocalized puncta ( $\left.\mu \mathrm{m}^{2}\right)$

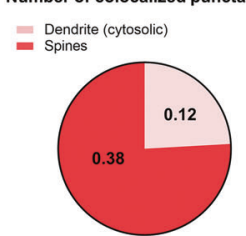

n

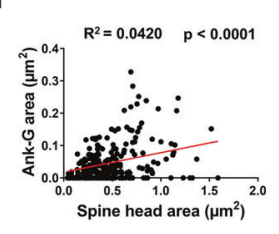

I Number of spines

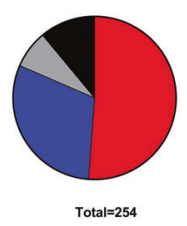

0

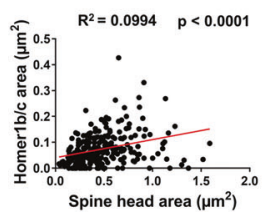

$j_{\text {AnkG }}$
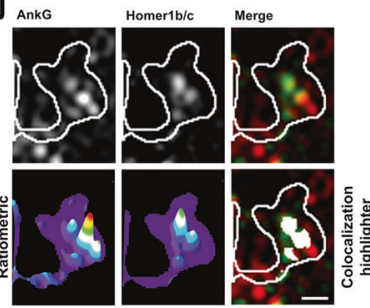

m

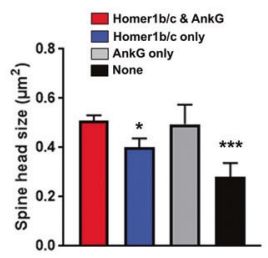

p

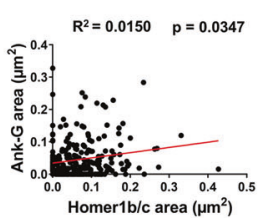

\section{PPXXF-mediated interaction of ankyrin-G and Homer1b/c regulates spine morphogenesis}

To understand interaction patterns of Homerlb/c and ankyrin-G in neurons, we expressed exogenous WT HAankyrin-G 190, or its mutants, P1606L, or F1609R, in mouse cultured cortical neurons. Comparing the effect of moderate exogenous expression of WT versus mutant forms of ankyrin-G 190 allows us to specifically test the effect of increased abundance of ankyrin-G 190 in spines, as shown to be induced by neuronal activity or by mood stabilizers, without altering the expression of all other isoforms that 
Fig. 2 Ankyrin-G 190 and Homer1b/c interaction in nanodomains within dendrites and spines. a Western blot of subcellular fractionation from 16-week-old mouse cortex is probed with ankyrin-G, Homer1b/c, $\mathrm{N}+/ \mathrm{K}+$ ATPase $\alpha 1$ (as a plasma membrane marker), PSD-95 (as a postsynaptic marker), and $\beta$-actin antibodies. Each lane was loaded with $10 \mu \mathrm{g}$ of each sample. WL whole cell lysate, S1 cytosol/membranes, S2 cytosol, P2 crude synaptosomes, PSD PSDenriched synaptosomal fraction. b Co-immunoprecipitation experiments with anti-ankyrin-G or anti-Homer1b/c from the PSD-enriched synaptosomal fraction. IgG control IgG, IP immunoprecipitation. c Binding of ankyrin-G and its truncation mutants to Homer1c. The top panel shows the immunoprecipitated full-length ankyrin-G or the truncated versions of ankyrin-G co-expressed with Homer1c. d Effects of point mutations in the PPXXF Homer-binding motif of ankyrin-G (P1606L and F1609R) on the interaction with Homer1c. The HEK293T cell lysate was analyzed by immunoblotting with HA or Flag antibody. $n=4$ per each group. $F(2,9)=24.10 ; F(2,9)=6.17$; ${ }^{*} p<0.05, * * * p<0.001$; followed by one-way ANOVA followed by a Bonferroni test. Data are represented as mean \pm SEM. e Schematic representation for the in situ proximity ligation assay (PLA). $\mathbf{f}$ Confocal images for detection of the interaction between HA-ankyrin-G wild-type (WT) or point mutants (red) and Flag-Homer1c (green) with PLA (magenta) in COS7 cells. Scale bar, $20 \mu \mathrm{m}$ (top and bottom). Bar graph of the PLA signal with the mutants of HA-ankyrin-G and FlagHomer1c. $n=13$ per each group. $F(2,36)=6.48 ;{ }^{*} p<0.05$, ${ }^{* *} p<$ 0.01; one-way ANOVA followed by a Bonferroni test. Data are represented as mean \pm SEM. g SIM image of a mCherry-expressing neuron to detect the interaction between ankyrin-G and Homer1b/c by PLA (cyan). Scale bar, $2 \mu \mathrm{m}$ (top panel). Zoomed images were shown from the boxed area in the top panel. Scale bar, $0.5 \mu \mathrm{m}$ (middle and botton panels). $\mathbf{h}$ Bar graph showing the PLA puncta ratio in spines versus dendrites, $n=9$ cells. i SIM image of mCherry-expressing neurons immunostained for anti-ankyrin-G (red) and anti-Homer1b/c (green). Scale bar, $2 \mu \mathrm{m}$. Colocalization is shown in white by 'colocalization highlighter' in ImageJ. $\mathbf{j}$ High-resolution image of boxed spine in i. Bottom panels: ratiometric images and colocalization (white). Scale bar, $0.5 \mu \mathrm{m}$. k Pie chart showing the highlighted puncta ratio of spines versus dendrites per spine area. 1 Pie chart of expression patterns of ankyrin-G and Homerlb/c in spines. m Spine head size from the chart $(\mathbf{k})$ was analyzed with a bar graph. $F(3,250)=5.77 ; * p$ $<0.05,{ }^{* * *} p<0.001$; one-way ANOVA followed by a Bonferroni test. Data are represented as mean \pm SEM. Correlation plot of the size of anti-ankyrin-G (n) or anti-Homer1b/c (o) nanodomains versus spine head area, and the size of anti-ankyrin-G versus anti-Homer1b/c nanodomains $(\mathbf{p}) . n=13$ cells. Head area: $0.452 \pm 0.017 \mu^{2}$; antiankyrin-G nanodomain area: $0.032 \pm 0.002 \mu \mathrm{m}^{2}$; anti-Homer $1 \mathrm{~b} / \mathrm{c}$ area: $0.056 \pm 0.003 \mu \mathrm{m}^{2}$.

may cause indirect effects on spines. The PLA method allows interacting proteins to be quantitatively and spatially visualized. SIM imaging of PLA signal revealed decreased Homer1 interaction with ankyrin- $\mathrm{G}^{\mathrm{P} 1606 \mathrm{~L}}$ and ankyrin$\mathrm{G}^{\mathrm{F} 1609 \mathrm{R}}$, as compared with WT ankyrin-G 190, in dendrites and spines (Fig. 3a, b). As shown before, exogenous expression of WT ankyrin-G 190 increased the spine head size. However, expression of the Homer1 interactiondeficient mutants of ankyrin-G 190 failed to induce a similar increase in spine head size, coincident with a decrease in PLA positive signal in spines (Fig. 3c; Supplementary Fig. 3). Interestingly, ankyrin-G ${ }^{\text {F1609R }}$ overexpression also increased the proportion of thin spines
(Fig. 3d, e). Total spine numbers were not altered in any of these conditions (Fig. 3d).

\section{The PPXXF motif in ankyrin-G 190 affects its mobile fraction in spines and spine head remodeling}

Our previous experiments demonstrated that HA-ankyrin$\mathrm{G}^{\mathrm{F} 1609 \mathrm{R}}$ significantly reduced the interaction with Homer1 and impaired the ability of ankyrin-G 190 to increase spine head size. We thus wanted to determine the impact of impairing ankyrin-G 190's interaction with Homer1 on its mobile fraction within spines. To this end, we performed fluorescence recovery after photobleaching (FRAP) timelapse imaging of GFP-ankyrin- $\mathrm{G}^{\mathrm{WT}}$ and GFP-ankyrin$\mathrm{G}^{\mathrm{F} 1609 \mathrm{R}}$ in dendritic spines of primary cortical neurons. Upon recovery after photobleaching for $200 \mathrm{~s}$, the mobile fraction of GFP-ankyrin-G $\mathrm{G}^{\mathrm{F} 1609 \mathrm{R}}$ was $22.44 \%$ higher than that of GFP-ankyrin-G ${ }^{\mathrm{WT}}$, indicating a higher mobile fraction for the Homer1 interaction-deficient mutant (Fig. 4a-c). This indicates that Homer1 interaction-deficient ankyrin-G 190 is less strongly associated with PSD or other stable components in spines that wild type ankyrin-G 190, likely due to its weak interactions with Homer1.

To assess the role of its interaction with Homer1 in ankyrin-G 190's ability to modulate spine head remodeling, we performed live confocal imaging of spines with mature morphologies, defined by head sizes above $0.6 \mu \mathrm{m}^{2}$. First, to visualize morphological remodeling of spine heads, we acquired stacks of images every minute for $10 \mathrm{~min}$ and generated $2 \mathrm{D}$ projections of spines for each time point, and used them to measure parameters reflecting spine head remodeling. While overexpression of GFP-ankyrin-G $\mathrm{G}^{\mathrm{WT}}$ caused an increase in perimeter dynamics, defined as the change in spine head perimeter during imaging, GFPankyrin-G ${ }^{\text {F1609R }}$ failed to do so (Fig. 4e, f; Movie 1). On the other hand, the area dynamics, defined as the change in the spine area, showed no significant difference between conditions (Fig. 4f). Finally, overexpression of GFP-ankyrin$\mathrm{G}^{\mathrm{WT}}$, but not GFP-ankyrin- $\mathrm{G}^{\mathrm{F} 1609 \mathrm{R}}$, caused an increase in morphological index dynamics, defined at the change in morphological index (perimeter ${ }^{2} / 4 \pi$ Area), which is an indicator of spine shape complexity (Fig. 4g). These data indicate that the PPXXF motif is important for ankyrin-G 190 's ability to form a stable pool in spines and to enhance spine head remodeling. We hypothesize that Homer1b/c acts to stabilize steady-state ankyrin-G pools in the spine head ultimately regulating spine head morphology.

\section{The PSD proteome is reshaped in HOMER1 KO mouse cortical synaptosomal membranes}

Ankyrin-G and Homer1b/c are reported to be enriched in the frontal cortex and hippocampus. Western blotting of 


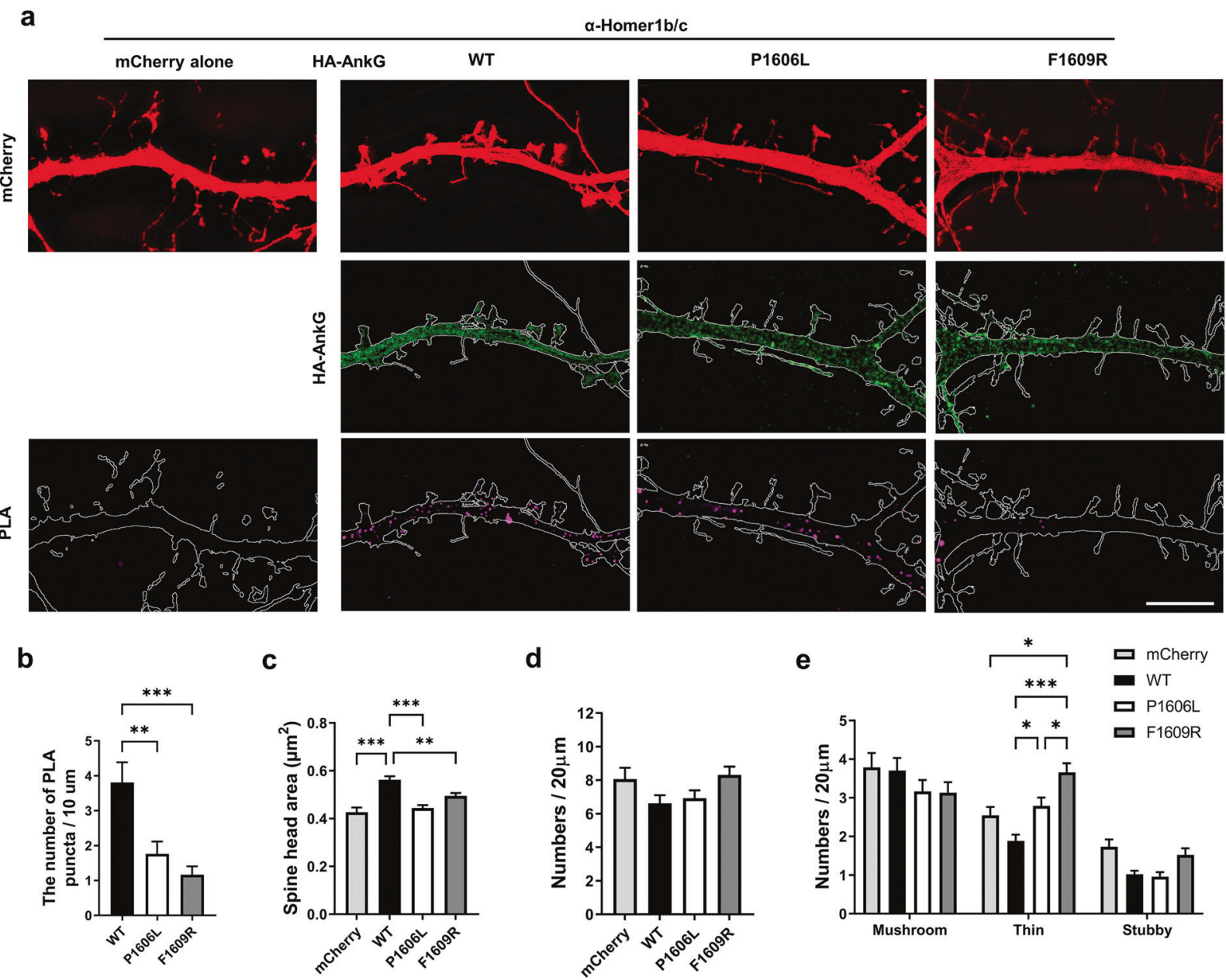

Fig. 3 Mutation of the PPXXF binding motif in ankyrin-G 190 attenuates its interaction with Homer1b/c in dendrites. a SIM images to detect the interaction between HA-ankyrin-G WT or mutants (P1606L or F1609R) and anti-Homer1b/c by in situ PLA. The PLA was conducted after transfection of HA-ankyrin-G WT or mutant constructs into the cultured mouse cortical neurons. Negative control was transfected with only mCherry construct. Scale bar, $2 \mu \mathrm{m}$. b The number of PLA puncta per $10 \mu \mathrm{m}$ from HA-ankryn-G WT, P1606L, and F1609R transfected neurons. PLA was performed with antiHomer1b/c and anti-HA antibodies. $F(2,123)=11.75$. c Spine head

Homerlb/c protein levels in the mouse cortex at different developmental time points revealed that Homerlb/c expression increases throughout postnatal development (Supplementary Fig. 4). Homer1b/c potentially forms an assembly platform and supports a structural framework for PSD proteins [18]. Our results show that the PPXXF motif in ankyrin$\mathrm{G}$ is critical to regulating the ankyrin- $\mathrm{G}$ dynamics in the spine heads (Fig. 4). We, therefore, hypothesized that the absence of Homer1 could affect the abundances of proteins in this specific cellular compartment. To assess the levels of ankyrin-G in $\mathrm{P} 2$ crude synaptosomal fractions in the presence $v s$. absence of Homer1, we assessed ankyrin-G by western blotting of P2 from WT and HOMERI KO mice. Unexpectedly, we found that ankyrin-G was more abundant in the size from mushroom spines was analyzed from only mCherry or HAankryn-G WT, P1606L, and F1609R transfected neurons with mCherry. $F(3,1445)=18.62$. d Total numbers of all spines were counted. $F(3,141)=3.64$. e Mushroom, thin, and stubby types of spine morphology were analyzed. $\mathrm{F}(6,423)=5.59 . n=13$ (only mCherry), 17 (WT), 19 (P1606L), 21 (F1609R) cells. * $p<0.05$; ** $p<$ $0.01 ; * * * p<0.001$; one-way ANOVA followed by a Bonferroni test for $\mathbf{b}-\mathbf{d}$ and two-way ANOVA followed by a Bonferroni test for $\mathbf{e}$. Data are represented as mean \pm SEM.

HOMER1 KO than in WT P2 fractions (Fig. 5a). In order to compare the abundance of synaptosomal membrane proteins in HOMER1 KO and WT mice, we performed TMT-LC/MS with P2 fractions (Fig. 5b). Differentially expressed proteins were quantified by comparing the normalized average reporter ion intensities of peptides among the biological replicates from HOMERI KO and WT mice. Proteome quantification was robust, and we were able to quantify 2937 proteins in both groups (Supplementary Table S2). 99 proteins showed significantly different abundance between HOMERI KO and WT $(p<0.05)$. Surprisingly, most $(87)$ of the 99 proteins increased in abundance in $\mathrm{P} 2$ fraction of HOMER1 KO mice, and only 12 were reduced compared to WT mice (Fig. 5c). Organizing the significantly altered proteins by functional 
Fig. 4 Mutation of the PPXXF binding motif in ankyrin-G 190 increases its mobile fraction in spines and reduces spine head dynamics. a Representative time-lapse of fluorescence recovery in a FRAP experiment in neurons overexpressing GFP-ankyrin-G 190 WT or F1609R. Scale bar, $1 \mu \mathrm{m}$. b Quantification of GFPankyrin-G or mutant

fluorescence intensity in each spine every $10 \mathrm{~s}$ for $200 \mathrm{~s}$. $F(20$, $126)=19.32$; two-way ANOVA followed by a Bonferroni test.

Data are represented as mean \pm SEM. $\mathbf{c}$ The graph of the mobile fraction (\% of post per pre bleaching at $200 \mathrm{~s}$ ) in GFPankyrin- $\mathrm{G}^{\mathrm{WT}}$ and GFP-ankyrin$\mathrm{G}^{\mathrm{F} 1609 \mathrm{R}}$-overexpressing neurons ( $n=7$ cells per each group, 3-5 spines from a neuron on three independent experiments). $\mathrm{t}(12)=4.24$; ***p $<0.001$; twotailed Student's $t$ test was performed. d Time-lapse imaging of spine head dynamics. Neurons were co-transfected with tdTomato together with GFP, GFP-ankyrin-G ${ }^{\mathrm{WT}}$ or GFPankyrin- $\mathrm{G}^{\mathrm{F} 1069 \mathrm{R}}$ and imaged for $10 \mathrm{~min}$. Seven representative images from 0 to $6 \mathrm{~min}$ taken at different time points were shown. e Analysis of perimeter dynamics from spine head. $F(2$, $48)=7.28$. f Quantification of area dynamics from spine head area. $F(2,48)=1.05$. g Spine morphological index (M.I. $=\mathrm{P}^{2} /$ $4 \pi \mathrm{A}$ ) dynamics in $10 \mathrm{~min}$ from spines. $F(2,48)=11.22$. GFP: $n=17$ spines, GFP-ankyrin$\mathrm{G}^{\mathrm{WT}}: n=16$ spines, GFPankyrin- $\mathrm{G}^{\mathrm{F} 1069 \mathrm{R}}: n=18$ spines; all spines were measured from 9 neurons per each group on four independent experiments. $* p<$ $0.05 ; * * p<0.01$; *** $p<0.001$; one-way ANOVA followed by a Bonferroni test. Data are represented as mean \pm SEM. See also Movie 1.
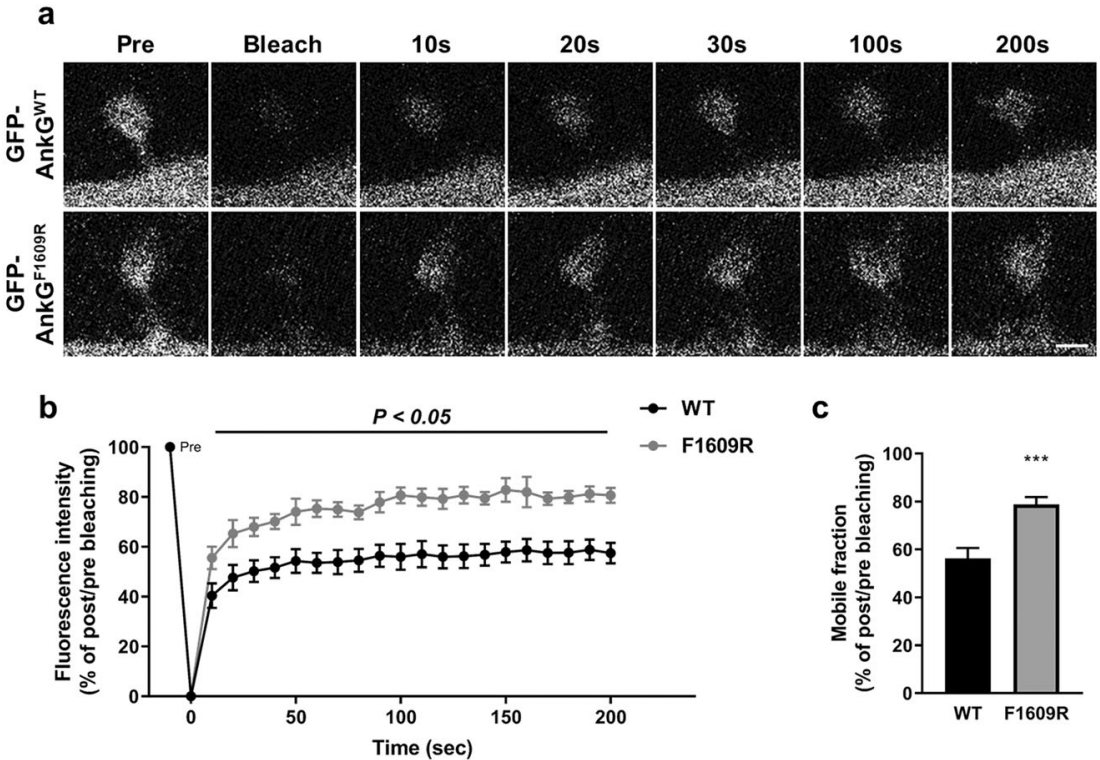

c

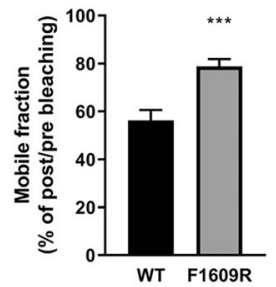

d

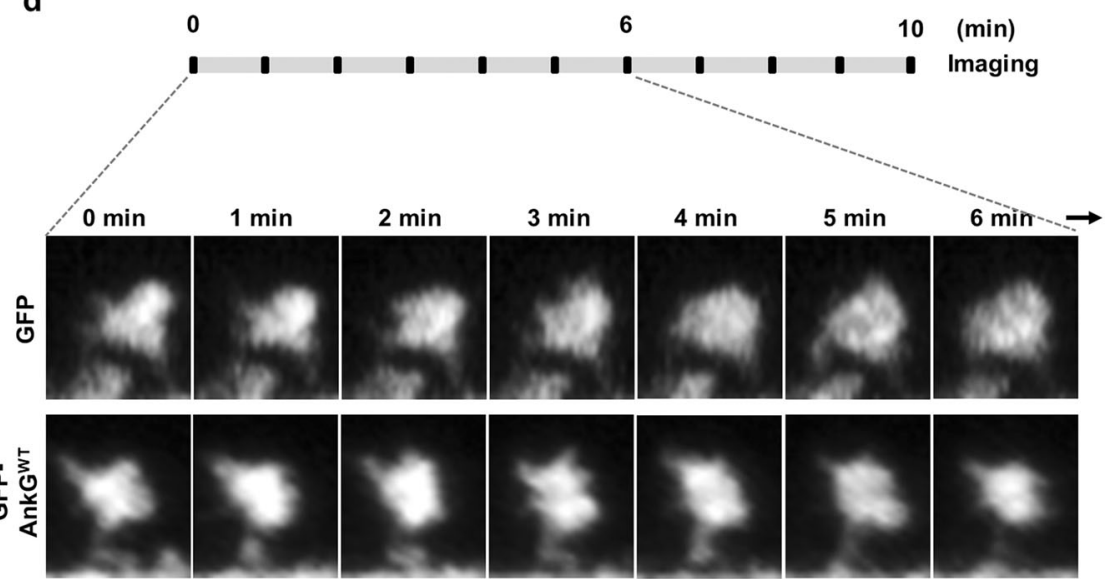

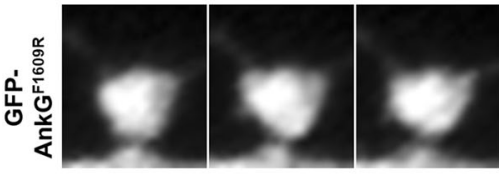

e

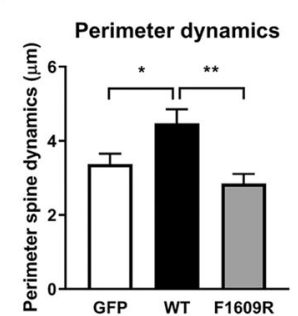

f

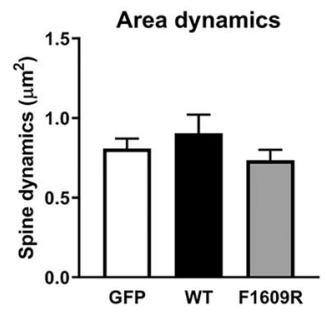

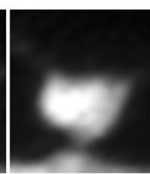

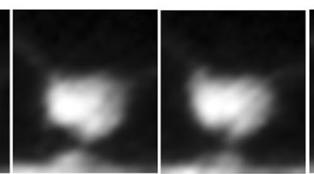

g
Morphological Index dynamics

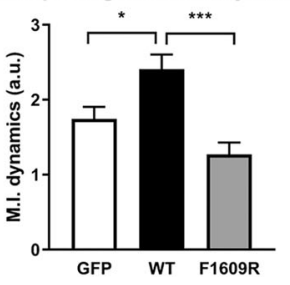

assignment, we found that the significantly altered proteins were involved in neuron projection, growth cone, myelin sheath, nuclear envelope, synapse, and cytoskeleton in the significantly enriched categories (Fig. 5d). In order to determine whether significantly altered proteins are enriched in PPXXF-containing proteins and PSDs, we retrieved all PPXXF-containing proteins and PSD proteins (Fig. 5e). We found a significant enrichment of PSD proteins $(n=39)$ in upregulated proteins (Fig. 5f); however, PPXXF-containing proteins were not significant within either the upregulated or downregulated proteins (Supplementary Fig. 5a).To understand how the absence of Homerl reshapes the protein network within the PSD, we seeded a network with the regulated proteins in PSD. The cutoff for regulated expression was 
a

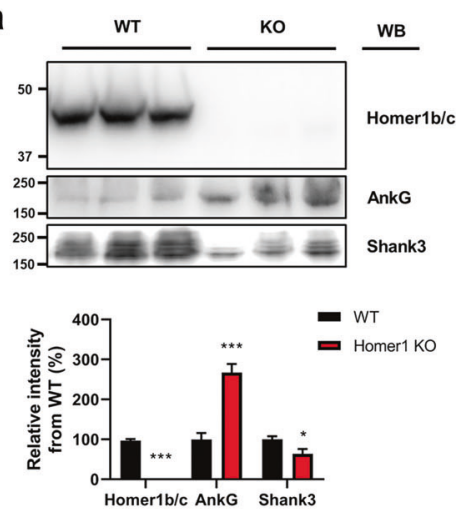

d

\begin{tabular}{ccc}
\hline Functional Annotation & $\boldsymbol{p}$ value & No. of Molecules \\
\hline Neuron projection & $7.00 \mathrm{E}-04$ & 12 \\
Growth cone & $5.11 \mathrm{E}-03$ & 7 \\
Myelin sheath & $4.09 \mathrm{E}-02$ & 6 \\
Nuclear envelope & $4.28 \mathrm{E}-02$ & 6 \\
Synapse & $4.28 \mathrm{E}-02$ & 9 \\
Cytoskeleton & $4.99 \mathrm{E}-02$ & 14 \\
\hline
\end{tabular}

b

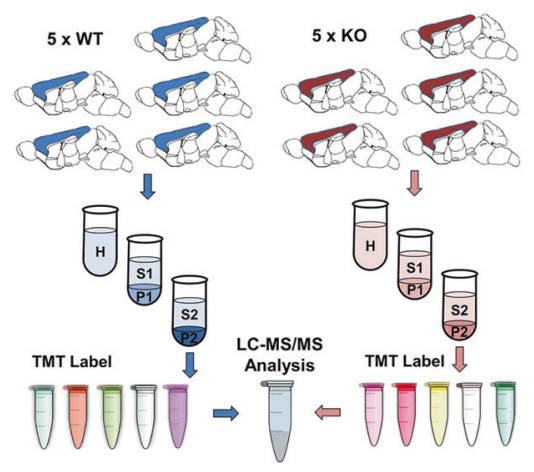

C

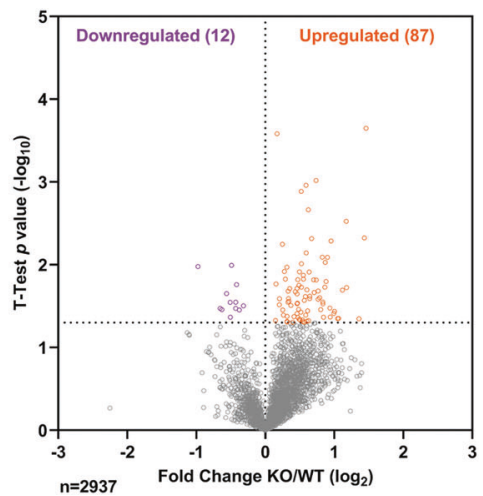

e

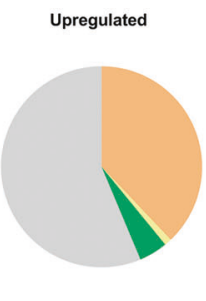

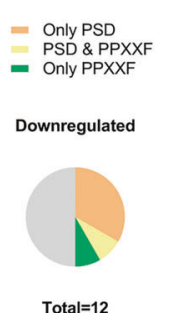

f Total detected proteins (2937)

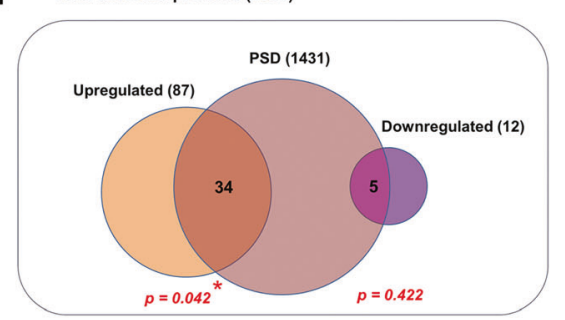

g

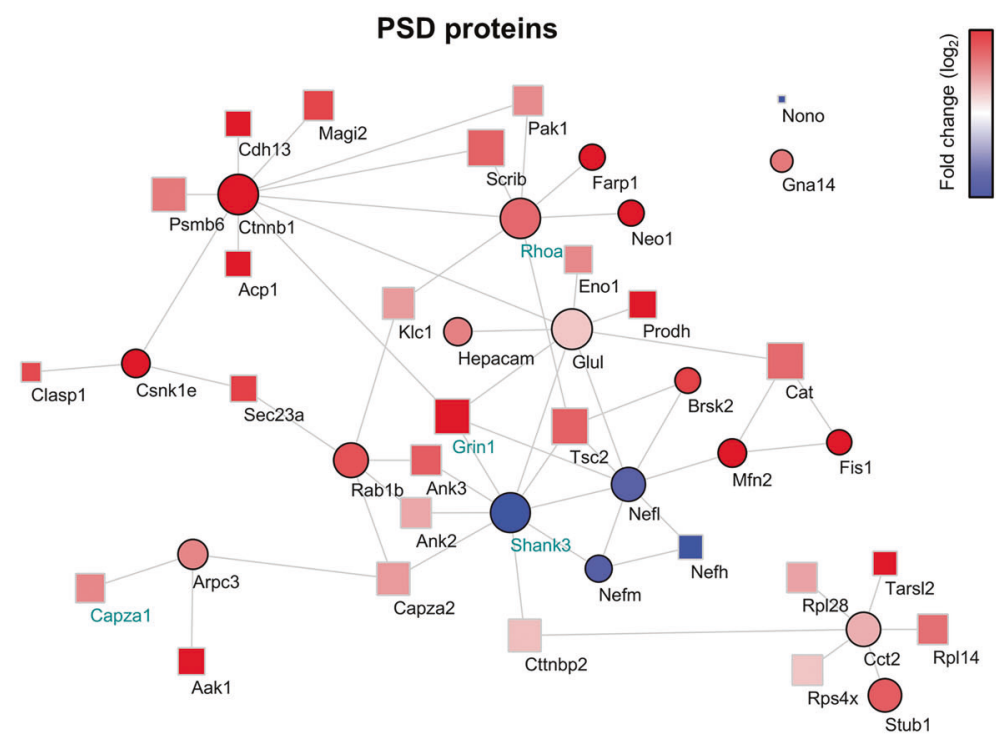

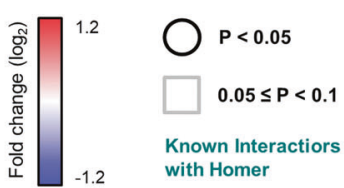

h

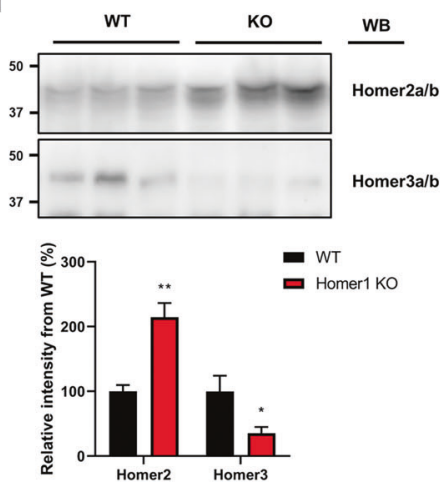

Fig. 5 The proteome is remodeled in the cortex of HOMERI KO mice. a Levels of Homerlb/c $(\mathrm{t}(8)=25.45)$, ankyrin-G $(\mathrm{t}(8)=6.32)$, and Shank3 $(\mathrm{t}(8)=2.54)$ proteins in $\mathrm{P} 2$ (crude synaptosomes) samples from the cortex of 3-week-old WT or HOMER1 KO mice. $n=5$ per each group. ${ }^{*} p<0.05$; ${ }^{* * *} p<0.001$; two-tailed Student's $t$ test was performed. Data are represented as mean \pm SEM. b Scheme of experimental work-flow of the proteomic analysis. c Volcano plot showing the fold change of individual protein levels versus significance between WT and HOMER1 KO mice. Significantly upregulated proteins are in orange $(p<0.05)$, significantly downregulated proteins are in purple $(p<0.05)$, and all other proteins are in gray. $\mathbf{d}$ Gene ontology (GO) analysis of statistically overrepresented biological processes among the significantly differentially regulated proteins in HOMER1 KO mice. The analysis was performed from data in $\mathbf{c}$ by DAVID. e Pie charts showing the presence in the PSD, inclusion of a PPXXF motif, or both, among the proteins significantly upregulated (left) and significantly downregulated (right) in HOMER1 KO versus WT mice. Gray indicates proteins that do belong to any of the categories. f Diagram showing the number of either upregulated or downregulated proteins presents in the PSD. Significance was tested by hypergeometric tests. $\mathrm{g}$ Cytoscape analysis of PSD proteins with altered levels coded by fold and significance of change. Circular nodes indicate $p<0.05$ and rectangular nodes indicate $0.05 \leq p<0.1$. Edges indicate predicted protein-protein interaction, including experimental data from the STRING database. The size of nodes indicates betweenness centrality. Previously known Homer1/2/3 interactors labeled in emerald. h Levels of Homer2a/b $(\mathrm{t}(8)=4.80)$ and Homer3a/ $\mathrm{b}(\mathrm{t}(8)=2.49)$ proteins in $\mathrm{P} 2$ samples from the cortex of 3 -week-old WT or HOMER1 KO mice. $n=5$ per each group. ${ }^{*} p<0.05$; ${ }^{*} p<$ 0.01 ; two-tailed Student's $t$ test was performed. Data are represented as mean \pm SEM. 
expanded to $p<0.1$ allowing the inclusion of many more known Homer interactors and facilitating interrogation of the PSD-PPI network within HOMERI KO mice. Interestingly, Shank3 and neurofilament proteins Nefh, Nefl, Nefm were nodes in a downregulated PPI subnetwork, with Shank3 having the highest centrality. Conversely, the downregulated subnetwork was much broader, with multiple nodes including RhoA, Ctnnb1, Grin1, Tsc2, and Ank3 $(0.05 \leq p<0.1)$ which were upregulated (Fig. 5g; Supplementary Fig. 6a). It is important to note that a significant increase of Homer2a/b abundances was observed in P2 samples, perhaps compensating for the decrease of Shank3 (Fig. 5h; Supplementary Fig. 6c). Taken together, our data suggest that Homerl is important for the maintenance of synaptic PPI networks, and its loss results primarily in the upregulation of synaptic abundance of postsynaptic and neurodevelopmental disorderrelated proteins.

\section{Discussion}

The PPXXF motif is known to mediate the interaction of Homer's EVH1 ligand motif with other proteins [16]. However, the global functions of these interactions are not known. Using computational approaches, we identified global roles for proteins containing the Homer1-interacting PPXXF motif within the postsynaptic compartment. PPXXF motif-containing proteins as a group are likely to be important in glutamatergic transmission and long-term potentiation, retrograde signaling, calcium channel function, protein transport, as well as in neurodevelopmental and psychiatric disease. Among the 150 PPXXF motifcontaining proteins in the PSD, ankyrin-G (ANK3), NR2A (GRIN2A), Shank2 (SHANK2), and Ca 1.2 (CACNAIC) are both BD and ASD risk factors. The enrichment of PSDPPXXF motif-containing proteins in neuropsychiatric disease risk factors suggests that proteins containing this motif may play a role in pathophysiological processes downstream of genetic risk factors. We found that the majority of PPXXF motif-containing psychiatric risk factors in PSDs are physically connected, and central hubs of this network are Cacna1c, Shank3, Grin2b, and ankyrin-G. While the interaction between Homer1 and Shank 2 or $\mathrm{Ca}_{\mathrm{V}} 1.2$ has already been reported $[39,40]$, future investigations of the interactions predicted by the presence of this motif is likely to reveal important biological and pathogenic pathways.

Ankyrin-G and Homer1 have emerged as candidate genes in multiple psychiatric disorders including BD, SZ, and ASDs [11-13, 41, 42]. These disorders share common synaptic pathologies, such as alterations in synaptic number and size. However, little is known about the underlying molecular mechanisms. Our findings demonstrate ankyrin$\mathrm{G}$ and Homer1, both neuropsychiatric disorder genes, interact in a complex to regulate spine morphology. This interaction reveals a potential mechanism through which Homer1 may regulate the localization and function of interacting proteins within the synapse. We identified two Homer recognition sites within ankyrin-G and showed a robust interaction between the $190 \mathrm{kDa}$ isoform of ankyrin$\mathrm{G}$ and Homer1b/c. This interaction was partially mediated by the Homer1 recognition motif (PPXXF) within the regulatory domain of ankyrin-G 190. It is possible that Homer1 also interacts with the giant ankyrin forms in other cellular locations and may be involved in additional physiological processes at those sites. These possibilities remain to be investigated in the future. We validated this interaction by in vivo and in vitro immunoprecipitation, PLA, and confirmed the interaction domain by site-directed mutagenesis. Because of the limited resolution of confocal microscopy, we utilized SIM to analyze the precise postsynaptic localization of ankyrin-G and Homerlb/c. High-resolution SIM allowed us to visualize and quantify the localization of ankyrin-G and Homer1b/c nanodomains in spine heads and revealed correlations between the presence of nanodomains and spine head size. While ankyrin-G colocalized with Homer1b/c in both the dendritic shaft and spine heads, colocalization was most extensive in spine heads. The presence of ankyrin-G alone, or ankyrin-G and Homer1b/c together predicted the largest spine head size, while the presence of Homer1b/c correlated with larger spine head sizes than spines without either protein.

Although colocalization supports a functional connection between proteins, in situ PLA confirms protein-protein interaction and allows spatial and quantitative visualization. To this end, we developed an approach combining PLA with SIM (PLA-SIM) to investigate the spatial organization of ankyrin-G-Homer1b/c interaction within spines and its relationship to spine architecture. The majority of interaction occurred in the dendritic shaft and spine head, but not the spine neck, although ankyrin-G is abundant there [28]. Point mutations in the PPXXF motif reduced the interaction in dendritic regions and led to attenuation of exogenous ankyrin-G 190-induced enlargement of spine head size. The effects of the mutations on protein interaction mirrored those on spine architecture. In our interpretation, exogenous expression of WT ankyrin-G 190 mimics its increased delivery into spines upon neuronal activity [28] or the mood stabilizer lithium [25]. Exogenous ankyrin-G 190 likely participates in multiprotein complexes or mesh-like matrix structures with Homer1 and Shank, promoting spine enlargement. On the other hand, while Homer1 interactiondeficient mutants of ankyrin-G 190 are delivered to dendrites and spines, they are unable to participate in such multiprotein matrices, failing to promote spine head enlargement. They may also disrupt Homer1-mediated arrays by competing with endogenous WT ankyrin-G 190. 
Remarkably, while exogenous expression of ankyrin-G 190 enhanced perimeter dynamics and morphological index dynamics in dendritic spines, this effect was abolished by the interaction-impairing point mutation in PPXXF. This suggests that a stable ankyrin-G pool within spines is essential for normal levels of spine head remodeling. We hypothesize that Homerlb/c stabilizes the steady-state levels of ankyrin-G within the spine head. Homer1 may thus provide a structural framework for other scaffolds within the PSD, such as ankyrin-G or Shank3, which in turn assemble downstream effector complexes, such as $\beta$-spectrin [28].

The unexpected finding that genetic deletion of all Homer1 isoforms causes an increase in ankyrin-G 190 abundance in synaptosomes, prompted us to perform a quantitative analysis of the cortical synaptosomal fraction proteome from Homer1 WT vs. KO mice. We found widespread proteomic dysregulation in HOMERI KO mouse synaptosomes, characterized primarily by dysregulation of postsynaptic proteins (39\% of total). This indicates that loss of Homer1 causes a global reshaping of the postsynaptic proteome and that Homer1 is important for the maintenance of correct synaptic proteome homeostasis. The proteome reshaping was surprisingly characterized by extensive upregulation of synaptic proteins, with significant enrichment of PSD proteins, suggesting perhaps a limiting role for Homer1 in postsynaptic protein networks. In addition to synaptic function, altered proteins also implicated unexpected processes like myelination and the nuclear envelope. The relationship of these with Homer1 is subject to future investigation.

Computational analysis through network seeding around dysregulated PSD proteins allows for the inclusion of proteins not detected by MS but known to interact with the seed proteins, and thus for higher predictive power. This approach indicated that Shank3 and neurofilaments are central nodes in the subnetwork of proteins downregulated in synaptosomes. As Shank3 had the highest centrality in this subnetwork and is known to interact with Homerl, downregulation of Shank3 consequent to the absence of Homer1 may have driven the downregulation of neurofilament proteins in synapses. This also suggests that Homer1 may function in stabilizing Shank3 at the synapse.

Conversely, we found that the subnetwork of proteins that increased in abundance in synaptic membranes was much broader, with multiple nodes including RhoA, Ctnnb1, Grin1, Tsc2, and Ank3. Of these, RhoA and ankyrin-G directly interact with Homer1, and their abundance in synaptic membranes may be limited by Homer1. Our proteomic data suggests that Homer1 is important for the maintenance of synaptic PPI networks, and its loss results primarily in the upregulation of synaptic abundance of postsynaptic and neurodevelopmental disorder-related proteins. Several mechanisms could potentially contribute to the synaptosomal proteome reshaping in HOMERI KO mice. Homer1 has been shown to drive the homeostatic downregulation of synaptic proteins and scaling down during sleep [43, 44]. Hence its absence may have the opposite effect, enabling the upregulation of synaptic proteins. Homer1 may also act as an architecturally limiting factor within the structure of the PSD. Homer and Shank form a postsynaptic polymeric network that stabilizes proteins at the PSD [18]. As for ankyrin-G, this may limit their diffusion and restrict their abundance in the PSD, while providing compartmentalized localization. In the absence of Homer1, this scaffold does not exist, proteins freely diffuse in an out of spines, and may be localized there due to other interacting partners, but not in a highly organized network. In addition, we found a robust upregulation of Homer2, which may partially compensate for the absence of Homer1. In the 3-week-old mouse brain Homer1b/c and Homer2a/b, but not Homer3a/b, are highly expressed in the cerebral cortex [45]. Homerla has also been shown to act as a dominant-negative form of Homer1 [46, 47]. In the HOMER1 KO mice, all isoforms of Homer1 are lost, including Homerla, potentially leading to effects opposite to its normal function in inhibiting synaptic transmission and spine morphogenesis. The absence of Homer 1 may also affect mGluR1/5-regulated protein translation [48]. Unexpectedly, the absence of Homer1 only affected the synaptosomal abundance of a few PPXXF-containing proteins. This suggests that the proteome alterations were unlikely to be globally driven by PPXXF domain proteins.

Our data also suggest that phenotypes in HOMERl KO mice $[14,15]$ may be in part caused by a global reshaping of the synaptosome. These data provide novel insights into a role for Homer1 in maintaining normal protein homeostasis in synapses and a potential role for ankyrin-G and Homer1 in neuropsychiatric disorder pathogenesis.

Acknowledgements This work was supported by R01MH107182 to PP and an Individual Biomedical Research Award from The Hartwell Foundation (J.N.S) to JS. We thank NU Nikon Cell Imaging Facility for the use of the N-SIM.

Author contributions From the Department of Physiology (SY, NP, LD, PP) and Department of Psychiatry and Behavioral Sciences (PP), and Department of Neurology (NK, JS) Northwestern University Feinberg School of Medicine, Chicago, Illinois. SY initiated the project and performed all experiments and data analysis unless otherwise stated. NP performed live imaging for spine dynamics and FRAP. NK and JS performed TMT-LC-MS. LD analyzed the data of PLA in situ immunocytochemistry. PP supervised the project and interpreted data.

\section{Compliance with ethical standards}

Conflict of interest The authors declare that they have no conflict of interest. 
Publisher's note Springer Nature remains neutral with regard to jurisdictional claims in published maps and institutional affiliations.

Open Access This article is licensed under a Creative Commons Attribution 4.0 International License, which permits use, sharing, adaptation, distribution and reproduction in any medium or format, as long as you give appropriate credit to the original author(s) and the source, provide a link to the Creative Commons license, and indicate if changes were made. The images or other third party material in this article are included in the article's Creative Commons license, unless indicated otherwise in a credit line to the material. If material is not included in the article's Creative Commons license and your intended use is not permitted by statutory regulation or exceeds the permitted use, you will need to obtain permission directly from the copyright holder. To view a copy of this license, visit http://creativecommons. org/licenses/by/4.0/.

\section{References}

1. Bosch M, Hayashi Y. Structural plasticity of dendritic spines. Curr Opin Neurobiol. 2012;22:383-8.

2. Glantz LA, Lewis DA. Decreased dendritic spine density on prefrontal cortical pyramidal neurons in schizophrenia. Arch Gen Psychiatry. 2000;57:65-73.

3. Hutsler JJ, Zhang H. Increased dendritic spine densities on cortical projection neurons in autism spectrum disorders. Brain Res. 2010;1309:83-94.

4. Irwin SA, Galvez R, Greenough WT. Dendritic spine structural anomalies in fragile-X mental retardation syndrome. Cereb Cortex. 2000;10:1038-44.

5. Konopaske GT, Lange N, Coyle JT, Benes FM. Prefrontal cortical dendritic spine pathology in schizophrenia and bipolar disorder. JAMA Psychiatry. 2014;71:1323-31.

6. Forrest MP, Parnell E, Penzes P. Dendritic structural plasticity and neuropsychiatric disease. Nat Rev Neurosci. 2018;19:215-34.

7. Penzes P, Cahill ME, Jones KA, VanLeeuwen JE, Woolfrey KM. Dendritic spine pathology in neuropsychiatric disorders. Nat Neurosci. 2011;14:285-93.

8. Brakeman PR, Lanahan AA, OBrien R, Roche K, Barnes CA, Huganir RL, et al. Homer: a protein that selectively binds metabotropic glutamate receptors. Nature. 1997;386:284-8.

9. Tu JC, Xiao B, Yuan JP, Lanahan AA, Leoffert K, Li M, et al. Homer binds a novel proline-rich motif and links group 1 metabotropic glutamate receptors with IP3 receptors. Neuron. 1998;21:717-26

10. Sala C, Piech V, Wilson NR, Passafaro M, Liu GS, Sheng M. Regulation of dendritic spine morphology and synaptic function by Shank and Homer. Neuron. 2001;31:115-30.

11. Kelleher RJ, Geigenmuller U, Hovhannisyan H, Trautman E, Pinard R, Rathmell B, et al. High-throughput sequencing of mGluR signaling pathway genes reveals enrichment of rare variants in autism. Plos ONE. 2012;7:e35003.

12. Norton N, Williams HJ, Williams NM, Spurlock G, Zammit S, Jones G, et al. Mutation screening of the Homer gene family and association analysis in schizophrenia. Am J Med Genet B. 2003;120b:18-21.

13. Rao ST, Lam MHB, Yeung VSY, Wing YK, Waye MMY. Association of HOMER1 rs2290639 with suicide attempts in Hong Kong Chinese and the potentially functional role of this polymorphism. Springerplus. 2016;5:767.

14. Jaubert PJ, Golub MS, Lo YY, Germann SL, Dehoff MH, Worley PF, et al. Complex, multimodal behavioral profile of the Homer1 knockout mouse. Genes Brain Behav. 2007;6:141-54.
15. Szumlinski KK, Lominac KD, Kleschen MJ, Oleson EB, Dehoff $\mathrm{MH}$, Schwarz MK, et al. Behavioral and neurochemical phenotyping of Homer1 mutant mice: possible relevance to schizophrenia. Genes Brain Behav. 2005;4:273-88.

16. Beneken J, Tu JC, Xiao B, Nuriya M, Yuan JP, Worley PF, et al. Structure of the Homer EVH1 domain-peptide complex reveals a new twist in polyproline recognition. Neuron. 2000;26:143-54.

17. Sun J, Tadokoro S, Imanaka T, Murakami SD, Nakamura M, Kashiwada K, et al. Isolation of PSD-Zip45, a novel Homer/vesl family protein containing leucine zipper motifs, from rat brain. Febs Lett. 1998;437:304-8.

18. Hayashi MK, Tang CY, Verpelli C, Narayanan R, Stearns MH, $\mathrm{Xu} \mathrm{RM}$, et al. The postsynaptic density proteins homer and shank form a polymeric network structure. Cell. 2009;137:159-71.

19. Gilman SR, Iossifov I, Levy D, Ronemus M, Wigler M, Vitkup D. Rare de novo variants associated with autism implicate a large functional network of genes involved in formation and function of synapses. Neuron. 2011;70:898-907.

20. Purcell SM, Moran JL, Fromer M, Ruderfer D, Solovieff N, Roussos $\mathrm{P}$, et al. A polygenic burden of rare disruptive mutations in schizophrenia. Nature. 2014;506:185-90.

21. Ferreira MA, O’Donovan MC, Meng YA, Jones IR, Ruderfer DM, Jones L, et al. Collaborative genome-wide association analysis supports a role for ANK3 and CACNA1C in bipolar disorder. Nat Genet. 2008;40:1056-8.

22. Schulze TG, Detera-Wadleigh SD, Akula N, Gupta A, Kassem L, Steele J, et al. Two variants in Ankyrin 3 (ANK3) are independent genetic risk factors for bipolar disorder. Mol Psychiatry. 2009;14:487-91.

23. Kordeli E, Lambert S, Bennett V. Ankyrin(G) - a new ankyrin gene with neural-specific isoforms localized at the axonal initial segment and node of ranvier. J Biol Chem. 1995;270:2352-9.

24. Bennett V, Healy J. Organizing the diseases fluid membrane bilayer: diseases linked to spectrin and ankyrin. Trends Mol Med. 2008;14:28-36.

25. Nanavati D, Austin DR, Catapano LA, Luckenbaugh DA, Dosemeci A, Manji HK, et al. The effects of chronic treatment with mood stabilizers on the rat hippocampal post-synaptic density proteome. J Neurochem. 2011;119:617-29.

26. Yoon S, Parnell E, Kasherman M, Forrest MP, Myczek K, Premarathne S, et al. Usp9X controls ankyrin-repeat domain protein homeostasis during dendritic spine development. Neuron. 2020;105:506-21.e507

27. Yoon S, Parnell E, Penzes P. TGF-beta-Induced phosphorylation of Usp9X stabilizes ankyrin-G and regulates dendritic spine development and maintenance. Cell Rep. 2020;31:107685.

28. Smith KR, Kopeikina KJ, Fawcett-Patel JM, Leaderbrand K, Gao $\mathrm{RQ}$, Schurmann B, et al. Psychiatric risk factor ANK3/Ankyrin-G nanodomains regulate the structure and function of glutamatergic synapses. Neuron. 2014;84:399-415.

29. Mohler PJ, Bennett V. Defects in ankyrin-based cellular pathways in metazoan physiology. Front Biosci. 2005;10:2832-40.

30. Rueckert EH, Barker D, Ruderfer D, Bergen SE, O'Dushlaine C, Luce CJ, et al. Cis-acting regulation of brain-specific ANK3 gene expression by a genetic variant associated with bipolar disorder. Mol Psychiatr. 2013;18:922-9.

31. Dedman A, McQuillin A, Kandaswamy R, Sharp S, Anjorin A, Gurling $H$. Sequencing of the ANKYRIN 3 gene (ANK3) encoding ankyrin $\mathrm{G}$ in bipolar disorder reveals a non-conservative amino acid change in a short isoform of ankyrin G. Am J Med Genet B Neuropsychiatr Genet. 2012;159B:328-35.

32. Hughes T, Hansson L, Sonderby IE, Athanasiu L, Zuber V, Tesli $\mathrm{M}$, et al. A loss-of-function variant in a minor isoform of ANK3 protects against bipolar disorder and schizophrenia. Biol Psychiatry. 2016;80:323-30. 
33. Hughes T, Sonderby IE, Polushina T, Hansson L, Holmgren A, Athanasiu L, et al. Elevated expression of a minor isoform of ANK3 is a risk factor for bipolar disorder. Transl Psychiat. 2018;8:210.

34. He Q, Arroyo ED, Smukowski SN, Xu J, Piochon C, Savas JN, et al. Critical period inhibition of NKCC1 rectifies synapse plasticity in the somatosensory cortex and restores adult tactile response maps in fragile $\mathrm{X}$ mice. Mol Psychiatry. 2019;24:1732-47.

35. Collins MO, Husi H, Yu L, Brandon JM, Anderson CN, Blackstock WP, et al. Molecular characterization and comparison of the components and multiprotein complexes in the postsynaptic proteome. $\mathbf{J}$ Neurochem. 2006;97:16-23.

36. Bayes A, Collins MO, Croning MD, van de Lagemaat LN, Choudhary JS, Grant SG. Comparative study of human and mouse postsynaptic proteomes finds high compositional conservation and abundance differences for key synaptic proteins. Plos ONE. 2012; 7:e46683.

37. Jung KM, Astarita G, Zhu C, Wallace M, Mackie K, Piomelli D. A key role for diacylglycerol lipase-alpha in metabotropic glutamate receptor-dependent endocannabinoid mobilization. Mol Pharm. 2007;72:612-21.

38. Trifilieff P, Rives ML, Urizar E, Piskorowski RA, Vishwasrao $\mathrm{HD}$, Castrillon J, et al. Detection of antigen interactions ex vivo by proximity ligation assay: endogenous dopamine $\mathrm{D} 2$-adenosine A2A receptor complexes in the striatum. Biotechniques. 2011;51: $111-8$.

39. Huang G, Kim JY, Dehoff M, Mizuno Y, Kamm KE, Worley PF, et al. $\mathrm{Ca}^{+}$signaling in microdomains - Homer1 mediates the interaction between RyR2 and Cav1.2 to regulate excitationcontraction coupling. J Biol Chem. 2007;282:14283-90.
40. Brandstatter JH, Dick O, Boeckers TM. The postsynaptic scaffold proteins ProSAP1/Shank2 and Homer1 are associated with glutamate receptor complexes at rat retinal synapses. J Comp Neurol. 2004;475:551-63.

41. Sanders SJ, Murtha MT, Gupta AR, Murdoch JD, Raubeson MJ, Willsey AJ, et al. De novo mutations revealed by whole-exome sequencing are strongly associated with autism. Nature. 2012;485:237-U124.

42. Iqbal Z, Vandeweyer G, van der Voet M, Waryah AM, Zahoor MY, Besseling JA, et al. Homozygous and heterozygous disruptions of ANK3: at the crossroads of neurodevelopmental and psychiatric disorders. Hum Mol Genet. 2013;22:1960-70.

43. Hu JH, Park JM, Park S, Xiao B, Dehoff MH, Kim S, et al. Homeostatic scaling requires group I mGluR activation mediated by Homer1a. Neuron. 2010;68:1128-42.

44. Diering GH, Nirujogi RS, Roth RH, Worley PF, Pandey A, Huganir RL. Homerla drives homeostatic scaling-down of excitatory synapses during sleep. Science. 2017;355:511-5.

45. Shiraishi-Yamaguchi Y, Furuichi T. The Homer family proteins. Genome Biol. 2007;8:206.

46. Sala C, Futai K, Yamamoto K, Worley PF, Hayashi Y, Sheng M. Inhibition of dendritic spine morphogenesis and synaptic transmission by activity-inducible protein homer1a. J Neurosci. 2003;23:6327-37.

47. Kammermeier PJ, Worley PF. Homer 1a uncouples metabotropic glutamate receptor 5 from postsynaptic effectors. P Natl Acad Sci USA. 2007;104:6055-60.

48. Guo WR, Molinaro G, Collins KA, Hays SA, Paylor R, Worley $\mathrm{PF}$, et al. Selective disruption of metabotropic glutamate receptor 5 -homer interactions mimics phenotypes of fragile $\mathrm{X}$ syndrome in mice. J Neurosci. 2016;36:2131-47. 